\title{
Análisis de las verbalizaciones desadaptativas del cliente y su relación con las verbalizaciones punitivas del terapeuta: un estudio de caso
}

\author{
María Xesús Froján Parga, Nerea Galván Domínguez, Isabel Izquierdo Alfaro, \\ Elena Ruiz Sancho y Carlos Marchena Giráldez \\ Universidad Autónoma de Madrid
}

ResUmen

En la Psicología en general, y en la Psicología Clínica en particular, se mantienen todo un conjunto de creencias inexactas sobre la aplicación del castigo, que se identifica únicamente con agresión física, sin tener en cuenta el potencial punitivo de la conducta verbal. De hecho, el castigo verbal es una herramienta ampliamente utilizada por el clínico para moldear la conducta verbal del cliente. Con el objetivo de clarificar el papel del castigo verbal en terapia, se analizó el presente caso. Se comenzó construyendo un sistema para categorizar las verbalizaciones desadaptativas del cliente y analizar su dinámica a lo largo de la terapia, encontrando una tendencia general descendente. Después se analizó la relación entre las verbalizaciones punitivas del terapeuta y las verbalizaciones del cliente, encontrando que el castigo apenas se aplicaba a las verbalizaciones desadaptativas del cliente, dirigiéndose sobre todo a otro tipo de verbalizaciones y a la propia conducta verbal.

Palabras clave: castigo, conducta verbal, proceso terapéutico, verbalizaciones desadaptativas.

\section{ABSTRACT}

In psychology in general, and in clinical psychology in particular, there remain a host of inaccurate beliefs about the application of punishment, which is only understood as physical aggression, without taking into account the punitive potential of verbal behavior. In fact, verbal punishment is a widely used tool by the clinician to shape client verbal behavior. In order to clarify the role of verbal punishment in therapy, the present case was analyzed. First, a system for categorizing the client's maladaptive utterances and analyzing their dynamic throughout therapy was constructed, finding a general downward trend. Subsequently, the relationship between the therapist's punitive verbalizations and the client's verbalizations was analyzed, finding that punishment was barely applied to the client's maladaptive verbalizations, mainly being addressed to other type of verbalizations and to the verbal behavior itself.

Keywords: punishment, verbal behavior, therapeutic process, maladaptive verbalizations.

Correspondencia: María Xesús Froján Parga. Departamento de Psicología Básica y de la Salud. c/. Ivan Pavlov, 6. Ciudad Universitaria de Cantoblanco. 28049 Madrid. Correo electrónico: mxesus.frojan@uam.es

Parte de este trabajo ha sido financiado por el Ministerio de Ciencia e Innovación en el marco del Plan Nacional de I+D+l 20082011 (Proyecto PSI2010-15908). 


\section{Introducción}

La Psicología ha mostrado una actitud claramente desfavorable hacia el estudio y la utilización del castigo en contextos aplicados, especialmente en los últimos 40 años. En éstos, el uso intencional y sistemático del castigo se entiende como un procedimiento a evitar, cuyo uso sólo está justificado en casos en los que ninguna otra alternativa es posible. Cuando se usa, el castigo se suele aplicar de forma intuitiva y no planificada. Así, por ejemplo, nos encontramos a menudo con psicólogos clínicos que rechazan enérgicamente el uso de cualquier tipo de castigo, para luego reprobar con la misma energía ciertas conductas de sus clientes en terapia, lo que constituye de hecho una forma de castigo. El problema principal es que la investigación sobre el castigo en ámbitos aplicados en general, y en el clínico en particular, es escasa, en ocasiones tendenciosa, generalmente inconsistente y difícil de interpretar de una forma clara y unificada.

Como venimos diciendo, las actitudes hacia el castigo generalmente son negativas y la conclusión más frecuente es que "el castigo debe usarse siempre como último recurso" (Martin y Pear, 1983). Si bien esta concepción se basa más en prejuicios que en datos empíricos, dada la escasez de investigaciones al respecto, lo cierto es que ha dado lugar a toda una serie de ideas ampliamente aceptadas, tanto por la población general como por los profesionales de la Psicología. Así, muy a grandes rasgos, el discurso que valora el castigo suele centrarse en lo siguiente:

1. El castigo tiene multitud de consecuencias indeseables, como conductas agresivas, estados emocionales negativos, supresión generalizada de las respuestas, ruptura de las relaciones sociales, conductas de escape o evitación, etc. cuya trascendencia y gravedad superan a las de las posibles ventajas derivadas de su aplicación.

2. El castigo es ineficaz en la supresión de conductas, puesto que la conducta castigada suele aumentar en los contextos en los que el castigo ya no está presente y/o ser remplazada por otra conducta inapropiada.

3. El castigo no sólo supone malestar y humillación para el que lo recibe, sino que suele afectar negativamente al que lo aplica, pudiendo convertirlo en alguien sádico, cruel e insensible al sufrimiento ajeno.

Pues bien, ninguna de estas afirmaciones caracteriza los resultados obtenidos con procedimientos de castigo adecuadamente administrados (Johnston, 1985). Esto no significa que todo lo recogido en los tres puntos anteriores no suceda cuando se utiliza el castigo, sino que no se trata de consecuencias inherentes al uso del mismo, que se den inevitablemente con todo tipo de castigos, en todas las situaciones y con todos los sujetos. Este trabajo surge como respuesta a esta situación, con el objetivo de contribuir al desarrollo de un conocimiento más profundo y riguroso sobre el proceso de castigo y, como se detallará más adelante, sobre su papel en la terapia de conducta. $Y$ es que la investigación acerca del castigo es necesaria para acabar con las creencias infundadas que predominan sobre él y para darle el lugar que le corresponde tanto en la investigación como en la práctica psicológica. 
Así pues, las razones que justifican el estudio del castigo y su uso en terapia de conducta pueden resumirse en los siguientes puntos (Lerman \& Vondran, 2002):

1) El castigo puede ser crítico para el éxito del tratamiento cuando las variables que mantiene la conducta no pueden ser identificadas o controladas.

2) El uso del castigo puede ser preferible al de otros procedimientos cuando, para evitar daños físicos, la conducta problema debe ser suprimida con rapidez. (Dura, 1991; Iwata et al., 1994; Vollmer \& Iwata, 1993).

3) Varios estudios apuntan a que los tratamientos derivados del análisis funcional pueden no ser eficaces en la reducción de la conducta a niveles clínicamente aceptables si no implican un componente de castigo (Grace, Kahng, \& Fisher, 1994; Hagopian, Fisher, Sullivan, Acquisto, \& LeBlanc, 1998; Wacker et al., 1990).

4) Mejorar nuestro conocimiento sobre el castigo también es importante porque es posible que tratamientos usados habitualmente en clínica y que se asocian a otros procesos reduzcan la conducta a través del mecanismo del castigo. Es decir, es posible que los mecanismos del castigo estén detrás de muchos de los tratamientos más populares diseñados a partir de un análisis funcional. De hecho, los hallazgos en investigación apuntan a que algunas de las variantes procedimentales de la extinción pueden actuar como castigo en lugar de, o en combinación con, la extinción (Lerman \& Iwata, 1996b; Mazaleski, Iwata, Rodgers, Vollmer, \& Zarcone, 1994)

5) La investigación básica muestra que diversas variables del refuerzo que mantiene la conducta influyen en los efectos directos e indirectos del castigo e interactúan con casi todas las variables que afectan a la respuesta durante la aplicación del mismo. Estas interacciones tienen implicaciones clínicas importantes, puesto que el uso del castigo es habitual cuando la relación respuesta-refuerzo no puede ser completamente anulada. En estos casos es de vital importancia tener en cuenta las interacciones entre el refuerzo y el castigo para optimizar los resultados del tratamiento.

Con todo ello, los estudios acerca del proceso de castigo, las variables con las que interactúa y los procedimientos que de él se derivan representan un número reducido de publicaciones, especialmente desde los años 70. Debemos tener en cuenta que el mundo investigador no es un mundo aislado e impermeable a las ideas y concepciones de la cultura en la que se inserta. Cada paso en una investigación, desde la elección del objeto de estudio y la forma de abordarlo hasta la determinación de sus implicaciones, depende de las actitudes predominantes en cada época y lugar. En los años 60 y 70, el surgimiento y expansión por EEUU y Europa Occidental de los movimientos sociales (pacifistas, defensores de los derechos civiles, ecologistas...) tuvo su reflejo en los temas y las prácticas investigadoras de la ciencia en general y de la Psicología en particular. La comunidad científica se vio obligada a hacer una reflexión profunda sobre las implicaciones éticas de sus investigaciones, lo que dio lugar a encendidos debates -muchos de los cuales aún hoy siguen abiertos- y a la idea de que "no todo vale" en ciencia. De este modo, se eliminaron de la práctica científica todos 
aquellos temas de estudio y procedimientos de investigación que implicasen algún riesgo para la integridad física y/o psicológica de los sujetos. Su estudio y aplicación sólo se justificaban en casos muy concretos, en los que los beneficios potenciales de la investigación superasen ampliamente a sus riesgos. En este contexto, las técnicas aversivas en general, y el castigo en particular, se convirtieron en los principales desterrados de la investigación en Psicología, que en una suerte de movimiento pendular se volcó en el estudio del refuerzo y los procedimientos que de éste se derivan. De esta forma llegamos al estado de la cuestión hoy en día, que no es otro que una alarmante falta de conocimiento riguroso acerca de qué es el castigo, cuáles son sus efectos, qué mecanismos implica, con qué variables interactúa y de qué modo lo hace. Como consecuencia, predominan toda una serie de prejuicios y actitudes hacia el castigo, carentes de base empírica pero ampliamente difundidos, que guían la actividad profesional de los psicólogos, ya sean investigadores, docentes o aplicados. De entre ellos destaca la identificación del castigo exclusivamente con el castigo físico y/o las conductas violentas, sin tener en cuenta el potencial poder punitivo de la conducta verbal. $Y$ es que la principal actividad que se lleva a cabo en terapia es, precisamente, la actividad verbal. El lenguaje es la principal herramienta del clínico para alcanzar sus objetivos, tanto los generales de la terapia (evaluar, explicar, intervenir), como los específicos de cada caso. En este sentido, cuando el terapeuta castiga en sesión lo hace a través del castigo verbal, que puede adoptar diversas formas en función de qué es lo que busque reducir o eliminar (una conducta concreta, un tipo de contenido verbal, una determinada forma de expresarse, el discurso del cliente en sí mismo...)

El presente trabajo se enmarca en la línea de investigación sobre la conducta verbal en contextos clínicos llevada a cabo por el grupo ACOVEO de la Universidad Autónoma de Madrid (Froján, Calero, \& Montaño, 2011; Froján, Montaño, \& Calero, 2006; Froján, Montaño, Calero, \& Ruiz, 2011; Froján, Pardo, Vargas, \& Linares, 2011) El objetivo principal de este equipo de investigación es estudiar los procesos que explican el cambio terapéutico a través del análisis de la interacción verbal en contextos clínicos, utilizando metodología observacional. En esta línea de investigación se considera que el paradigma conductual es la mejor alternativa para realizar una aproximación científica al estudio del comportamiento, específicamente en cuanto a la propuesta de la interacción terapeuta-cliente como un proceso de moldeamiento, a la explicación del lenguaje observado en la clínica por los principios de condicionamiento clásico y operante y a la conceptualización de la terapia de conducta como la aplicación de operaciones conductuales básicas para el tratamiento de problemas psicológicos ${ }^{1}$. Enmarcado en este contexto, el presente trabajo pretende contribuir al desarrollo de un cuerpo de conocimiento riguroso sobre el proceso de castigo y servir de punto de partida para futuras investigaciones en el área. Más concretamente, nuestro objetivo

1 Remitimos al lector interesado a la página web del grupo ACOVEO (http://www.grupoacoveo.com/), donde podrá encontrar información más detallada sobre su actividad, así como algunos de los artículos que ha publicado. 
principal es describir y analizar la relación existente entre las verbalizaciones del cliente y las verbalizaciones punitivas del terapeuta. Para ello nos hemos planteado comenzar estudiando, por un lado, cómo se van modificando cierta clase de verbalizaciones del cliente a lo largo de la terapia y, por otro lado, ante qué tipo de verbalizaciones del cliente aplica el castigo el terapeuta. Así pues, se trata sólo del primer paso en una línea que pretende analizar cómo se utiliza el castigo en el contexto terapéutico, qué verbalizaciones del cliente se castigan y cuáles no y los efectos que tiene su aplicación en el desarrollo de la terapia.

\section{Método}

El presente estudio parte del trabajo llevado a cabo por Ruiz, Froján y Calero (en prensa) acerca de la interacción verbal entre terapeuta y cliente durante el desarrollo de la terapia. En dicho trabajo se desarrolló el Sistema de Categorización de la Interacción de la Conducta Verbal en Terapia (SISC- INTER- CVT) para analizar la relación existente entre las verbalizaciones del terapeuta y el cliente a lo largo de la terapia. Una de las hipótesis de partida en dicho estudio era que las verbalizaciones del cliente incluidas en el grupo de "anti terapéuticas" irían seguidas de verbalizaciones del terapeuta categorizadas como "Función de castigo". Si bien la hipótesis se cumple, un análisis detallado de los resultados mostró que las verbalizaciones categorizadas como Fracaso y Malestar no sólo no disminuían al final de la terapia sino que incluso se incrementaban. La autora proponía varias razones para explicar este aumento: el desarrollo de dependencia respec- to a la terapia en las últimas fases de ésta, la competencia que supone el refuerzo externo de las verbalizaciones castigadas en terapia y la falta de sistematicidad en la aplicación del castigo por parte del terapeuta. A la luz de este hecho nos interesamos por la relación entre las verbalizaciones desadaptativas del cliente y las verbalizaciones punitivas del terapeuta. Así mismo, nos basamos en la metodología de investigación del citado estudio para hacer un primer acercamiento al análisis de esa relación.

Participantes:

Se analizó el caso clínico de N. integrado por 10 sesiones de una duración aproximada de una hora. La intervención la llevó a cabo una terapeuta experta con más de 15 años de experiencia en la práctica clínica, en el Instituto Terapéutico de Madrid (ITEMA), un centro privado de terapia cognitivo-conductual. El cliente, mujer de 32 años de edad, recibió un tratamiento individual centrado en los problemas de pareja presentes en aquel momento. El proceso de intervención finalizó con el alta terapéutica.

De acuerdo con lo expuesto en el Código Deontológico del Psicólogo (arts. 40 y 41), la grabación y cesión para el presente estudio cursó con el consentimiento informado del cliente, el terapeuta y la directora del centro.

\section{Instrumentos:}

La grabación de los casos se realizó a través de un circuito cerrado de cámaras. En orden de preservar el anonimato del cliente, la imagen sólo mostraba la figura del terapeuta.

El software utilizado para la observación y el registro de las sesiones fue la versión 6.0 de The 
Observer XT, comercializado por Noldus Information Technology, mientras que para el análisis de la fiabilidad intejueces de los registros se utilizó la versión 7.0 de este mismo programa.

\section{Procedimiento:}

El desarrollo del presente trabajo puede dividirse en las siguientes fases:

1) Establecimiento de la unidad de categorización. Se acordó registrar la conducta verbal manifiesta emitida por el cliente en cada una de las sesiones clínicas, en interacción con el terapeuta.

2) Observación no sistemática y propuestas iniciales de categorización. Se comenzó a construir un sistema de categorización preliminar en el que recoger aquellas verbalizaciones del cliente consideradas contrarias a los objetivos terapéuticos. Tres jueces observaron y registraron informalmente dichas verbalizaciones en diversos fragmentos de la terapia, planteando después distintas propuestas para su organización y categorización. Se analizó y discutió cada propuesta y, contando con el asesoramiento de dos psicólogas expertas, se llegó a una primera versión del sistema de categorización.

3) Depuración y propuesta definitiva del sistema de categorización. El sistema inicial fue puesto a prueba, mostrando numerosos problemas en la definición de las distintas categorías. Comenzó entonces un proceso de depuración profunda del sistema inicial mediante su aplicación, discusión y corrección, hasta dar lugar a un sistema definitivo que superase los distintos problemas que se fueron detectando.

4) Observación sistemática y análisis del grado de acuerdo interjueces. A continuación se procedió al registro sistemático de las verbalizaciones desadaptativas del cliente con la ayuda del programa informático The Observer $X T$ versión 6.0. Dicha tarea fue llevada a cabo por dos de las observadoras implicadas en la construcción del sistema de categorías, tras consensuar unos criterios de categorización claros. A continuación se procedió al cálculo de la fiabilidad interjueces, mediante la comparación de los registros de tres de las diez sesiones que componen el caso.

5) Análisis de la evolución de las verbalizaciones desadaptativas del cliente. Una vez registradas las verbalizaciones desadaptativas, se procedió al análisis de su dinámica a lo largo de la terapia, tanto en términos totales como distribuidas en cada una de las categorías contempladas en el sistema de categorización.

6) Análisis preliminar de la relación entre las verbalizaciones punitivas del terapeuta y las verbalizaciones del cliente. Finalmente, se llevó a cabo un análisis exploratorio de la relación entre las verbalizaciones del terapeuta que podían ser categorizadas como de castigo y las verbalizaciones del cliente. El objetivo de este análisis fue determinar qué tipo de verbalizaciones del cliente castiga el terapeuta en sesión y si se trata de verbalizaciones desadaptativas, tal y como nosotros las definimos, o si se trata de otro tipo de verbalizaciones. Durante este análisis, hubo que incluir dos nuevas categorías de la conducta verbal del cliente -Discurso y Otras Verbalizaciones-, para dar cabida a las verbalizaciones del cliente que iban seguidas de castigos del terapeuta, pero no podían incluirse en ninguna de las categorías contempladas en el sistema previamente desarrollado. 


\section{Resultados}

Sistema de categorías de la conducta verbal desadaptativa del cliente (SISC-DESAD-CVC):

A continuación se recoge el sistema de categorías desarrollado para registrar las conductas verbales desadaptativas del cliente atendiendo a su contenido. Se consideraron verbalizaciones desadaptativas todas aquellas expresiones de malestar, dificultad o incapacidad del cliente con respecto a las distintas áreas problema identificadas durante la terapia.

Evolución de las verbalizaciones desadaptativas a lo largo de la terapia:

A continuación presentamos los resultados obtenidos del registro de las verbalizaciones desadaptativas del cliente a los largo de las diez sesiones de terapia. Los resultados se presentan en tanto en verbalizaciones totales como organizados según las categorías del SISC-DESAD-CVC
Como es de esperar, las verbalizaciones desadaptativas del cliente siguieron una tendencia general descendente a lo largo de la terapia, con altibajos puntuales en función de la fase concreta en la que nos situemos. Así, los porcentajes de verbalizaciones desadaptativas más altos, de entre el $15 \%$ y el $20 \%$, se encontraron durante las sesiones de evaluación (uno a tres), en las que el cliente da cuenta de sus problemas, y en las sesiones de tratamiento (cinco a nueve), en las que el cliente expresa sus dificultades durante la realización de las distintas tareas propuestas por el terapeuta. Los porcentajes más bajos, en torno al $2 \%$, se encontraron durante la explicación del análisis funcional (sesión cuatro), puesto que en esta sesión el protagonista es el terapeuta y el cliente apenas habla, y en la fase de consolidación de los cambios (sesión diez).

Tabla 1

Categorías incluidas en el SISC-DESAD-CVC

Sistema de categorización de la conducta verbal desadaptativa del cliente (SISC-DESAD-CVC)

$\begin{array}{ll}\text { Inespecífico } & \begin{array}{l}\text { Verbalizaciones desadaptativas del cliente acerca de aspectos no centrales en la terapia y que no } \\ \text { pueden considerarse dentro de los objetivos de tratamiento }\end{array} \\ \text { Familia } & \begin{array}{l}\text { Verbalizaciones desadaptativas del cliente sobre aspectos de la relación que mantiene con su } \\ \text { propia familia }\end{array} \\ \text { Pareja Cliente } & \text { Verbalizaciones desadaptativas del cliente acerca de su propia conducta en la relación de pareja } \\ \text { Pareja (otro) } & \begin{array}{l}\text { Verbalizaciones desadaptativas del cliente acerca de la conducta de su compañero en la relación } \\ \text { conducta }\end{array} \\ \text { de pareja } \\ \begin{array}{l}\text { Pareja (otro) } \\ \text { familia }\end{array}\end{array}$




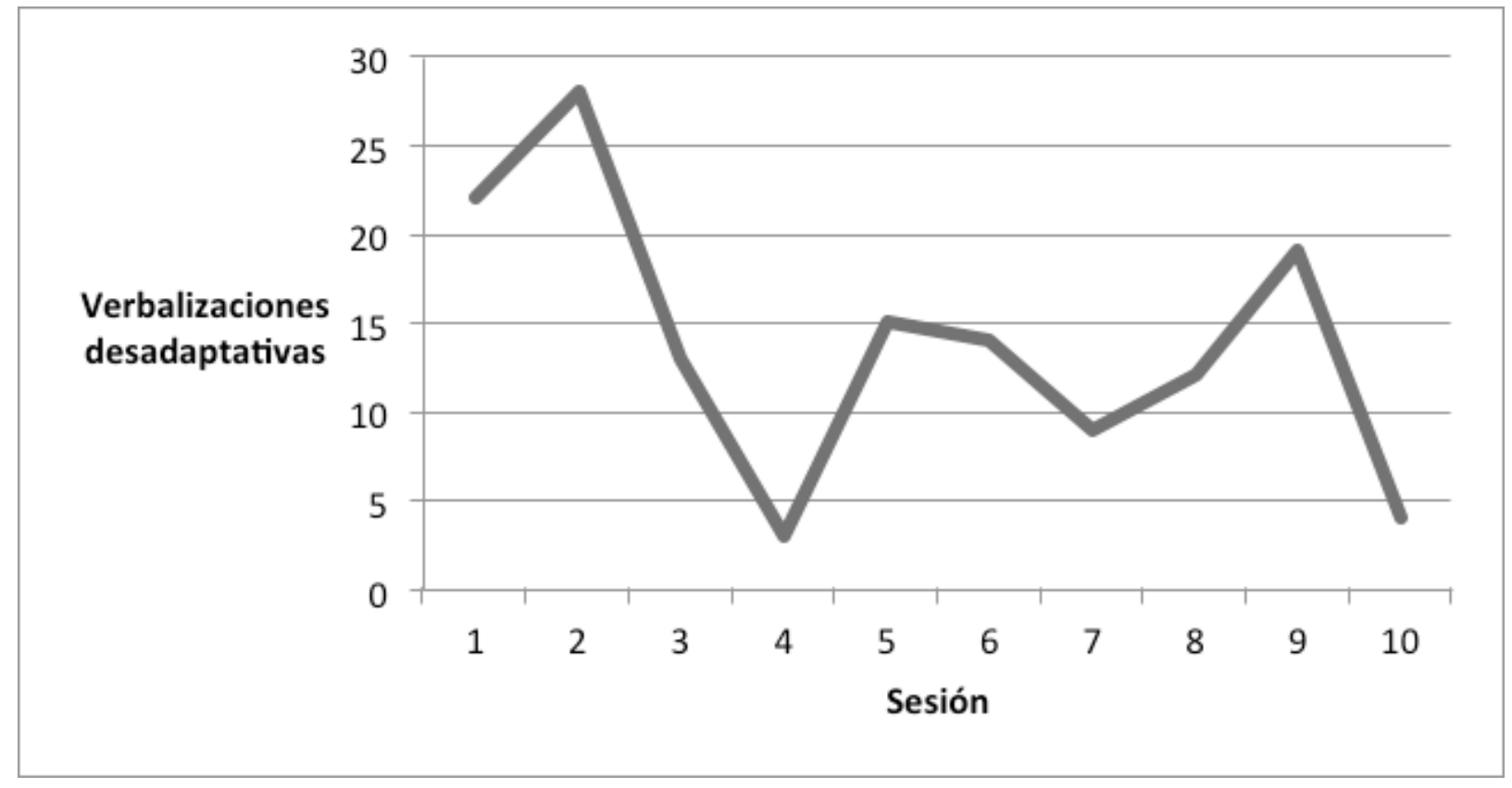

Figura 1. Verbalizaciones desadaptativas del cliente en totales (Frecuencias absolutas).

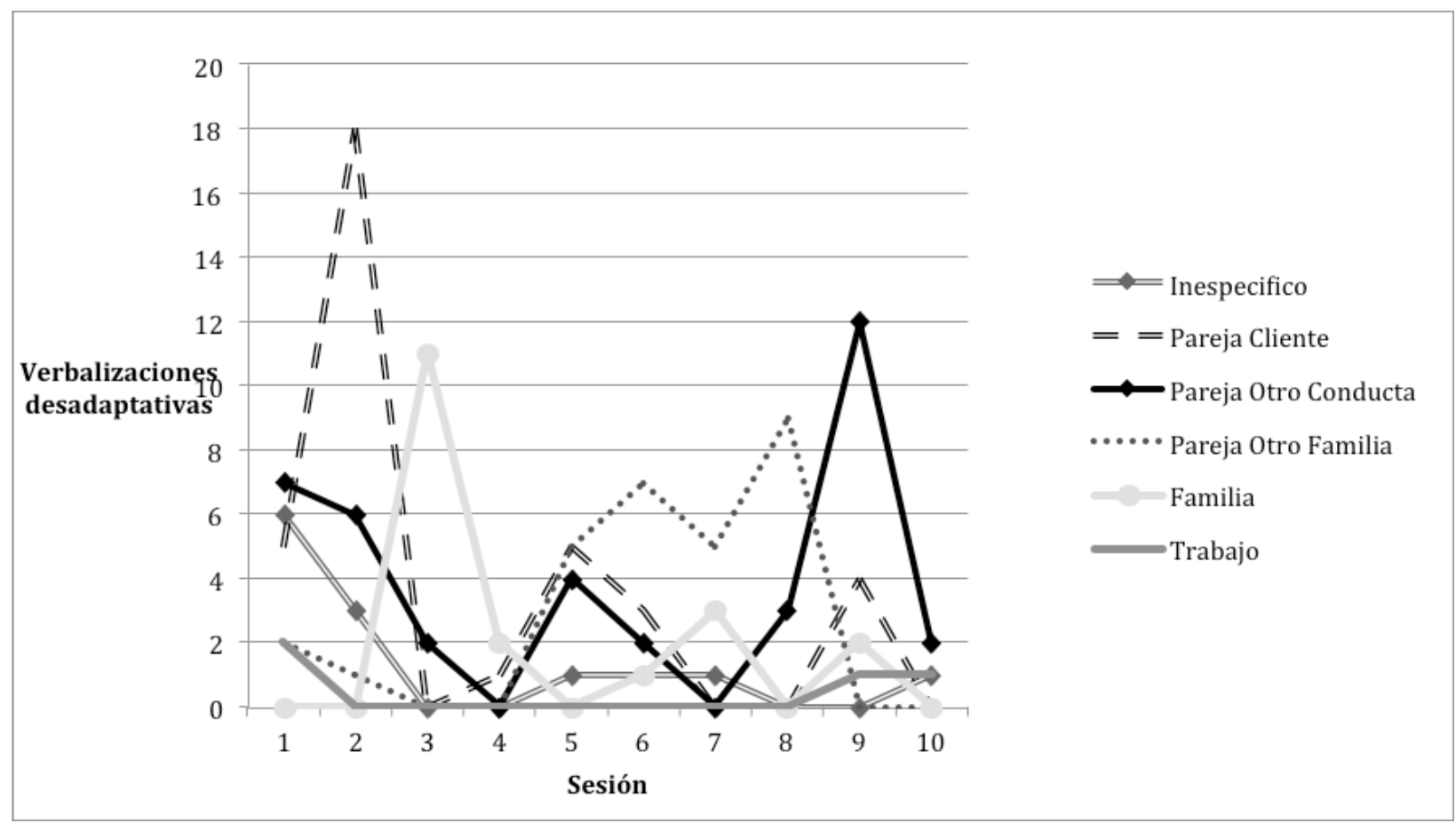

Figura 2. Verbalizaciones desadaptativas de cliente por categorías, según el SISC-DESAD-CVC (Frecuencias absolutas). 
Relación entre las verbalizaciones punitivas del terapeuta y las verbalizaciones del cliente:

Para definir qué verbalizaciones del terapeuta podían categorizarse como de castigo, se utilizó el SISC-INTER-CVT (Ruiz, 2011), un sistema de categorización de la conducta verbal de cliente y terapeuta desarrollado recientemente por nuestro grupo de investigación. En este sistema, la categoría Castigo se define como aquella "verbalización del terapeuta que, interrumpiendo o no la verbalización del cliente, muestra desaprobación, rechazo y/o no aceptación de la conducta emitida por éste" (Ruiz, 2011) Estas verbalizaciones del terapeuta pueden expresar desacuerdo con el contenido de las verbalizaciones del cliente, pueden ir dirigidas a interrumpir o evitar su discurso o ambas cosas a la vez.

Ejemplos:

- Cliente: "Bueno, le puedo decir: estoy ahí, si necesitas algo me llamas, ya lo sabes"

- Psicólogo: "Si, pero hay formas y formas de decirlo. No así, como si fueses una guía telefóni$c a^{\prime \prime}$ (Función de castigo dirigida al contenido expresado por el cliente)

- Cliente: "Si, pero fue una mezcla de bien, porque me siento bien por haber sido capaz de hacer esto, pero mal porque me, me... pues no sé, me era un desprecio más que no me gustó nada"

- Psicólogo: "No, no, espera, espera, espera" (Función de castigo dirigida a interrumpir el discurso del cliente)

Con la ayuda de los registros de la conducta verbal del terapeuta realizados previamente por nuestro equipo mediante el SISC-INTER-CVT, se buscaron todas las verbalizaciones del terapeuta que podían categorizarse como de castigo. A continuación, se localizaron las verbalizaciones del cliente que precedían a los castigos del terapeuta, con el objetivo de determinar qué tipo de verbalizaciones del cliente castiga el terapeuta en sesión y si se trata de verbalizaciones desadaptativas, tal y como nosotros las hemos definido, o si se trata de otro tipo de verbalizaciones. En este sentido, a la hora de clasificar las verbalizaciones del cliente que precedían a los castigos del terapeuta, se utilizaron las categorías del SISC-DESAD-CVC, así como dos categorías nuevas: Discurso y Otras Verbalizaciones. La categoría Discurso incluye todas aquellas ocasiones en las que el cliente interrumpe al terapeuta, no para de hablar, se desvía del tema o no contesta a lo que se le pregunta, de tal forma que el terapeuta castiga su conducta verbal en sí misma. Por su parte, la categoría Otras Verbalizaciones abarca todas aquellas verbalizaciones del cliente que son castigadas por el terapeuta y que no se incluyen en ninguna de las categorías del SISC-DESAD-CVC ni tampoco pueden incluirse bajo la etiqueta Discurso.

A continuación presentamos los resultados obtenidos del análisis exploratorio de la relación entre las verbalizaciones punitivas y del terapeuta las verbalizaciones del cliente.

Como refleja la Tabla 2, la categoría que precedió a un castigo del terapeuta en más ocasiones fue, con diferencia, Otras Verbalizaciones. Así, del total de castigos emitidos por el terapeuta, un $75,9 \%$ iban precedidos de una verbalización del cliente que no puede incluirse dentro de ninguna de las categorías 
Tabla 2

Verbalizaciones del cliente seguidas de verbalizaciones de castigo por parte del terapeuta

\begin{tabular}{lrrrrrrrrrrrr}
\hline & S1 & S2 & S3 & S4 & S5 & S6 & S7 & S8 & S9 & S10 $\begin{array}{c}\text { Total } \\
\text { verbaliza } \\
\text { castigadas }\end{array}$ \\
\hline Inespecífico & 1 & 0 & 0 & 0 & 0 & 0 & 0 & 0 & 0 & 0 & 1 \\
Pareja Cliente & 0 & 0 & 0 & 0 & 0 & 0 & 0 & 0 & 0 & 0 & 0 \\
Pareja Otro Conducta & 0 & 1 & 0 & 0 & 0 & 1 & 0 & 0 & 0 & 0 & 2 \\
Pareja Otro Familia & 0 & 0 & 0 & 0 & 0 & 0 & 1 & 0 & 0 & 0 & 1 \\
Familia & 0 & 0 & 0 & 0 & 0 & 0 & 0 & 0 & 0 & 0 & 0 \\
Trabajo & 0 & 0 & 0 & 0 & 0 & 0 & 0 & 0 & 0 & 0 & 0 \\
Discurso & 2 & 2 & 1 & 0 & 4 & 1 & 1 & 2 & 3 & 0 & 16 \\
Otras verbalizaciones & 5 & 1 & 2 & 1 & 8 & 12 & 14 & 3 & 11 & 6 & 63 \\
Total castigos & 8 & 4 & 3 & 1 & 12 & 14 & 16 & 5 & 14 & 6 & 83 \\
\hline
\end{tabular}

del SISC-DESAD-CVC ni tampoco dentro de la categoría Discurso. La siguiente categoría que más apareció seguida de castigos fue Discurso, un $19,2 \%$ de la veces. Finalmente las siguieron, muy de lejos, las categorías del SISC-DESADCVC Pareja Otro Conducta (2,4\%), Inespecífico (1,2\%) y Pareja Otro Familia (1,2\%).

Por otra parte, también podemos observar cómo evolucionaron las verbalizaciones de castigo a lo largo de las diez sesiones que duró la terapia. Como es de esperar, las sesiones correspondientes al tratamiento mostraron una mayor frecuencia de verbalizaciones de castigo, observándose una disminución de éstas en las últimas sesiones.

En la Figura 3 podemos ver la evolución a lo largo de la terapia de las verbalizaciones de castigo del terapeuta -según la definición del

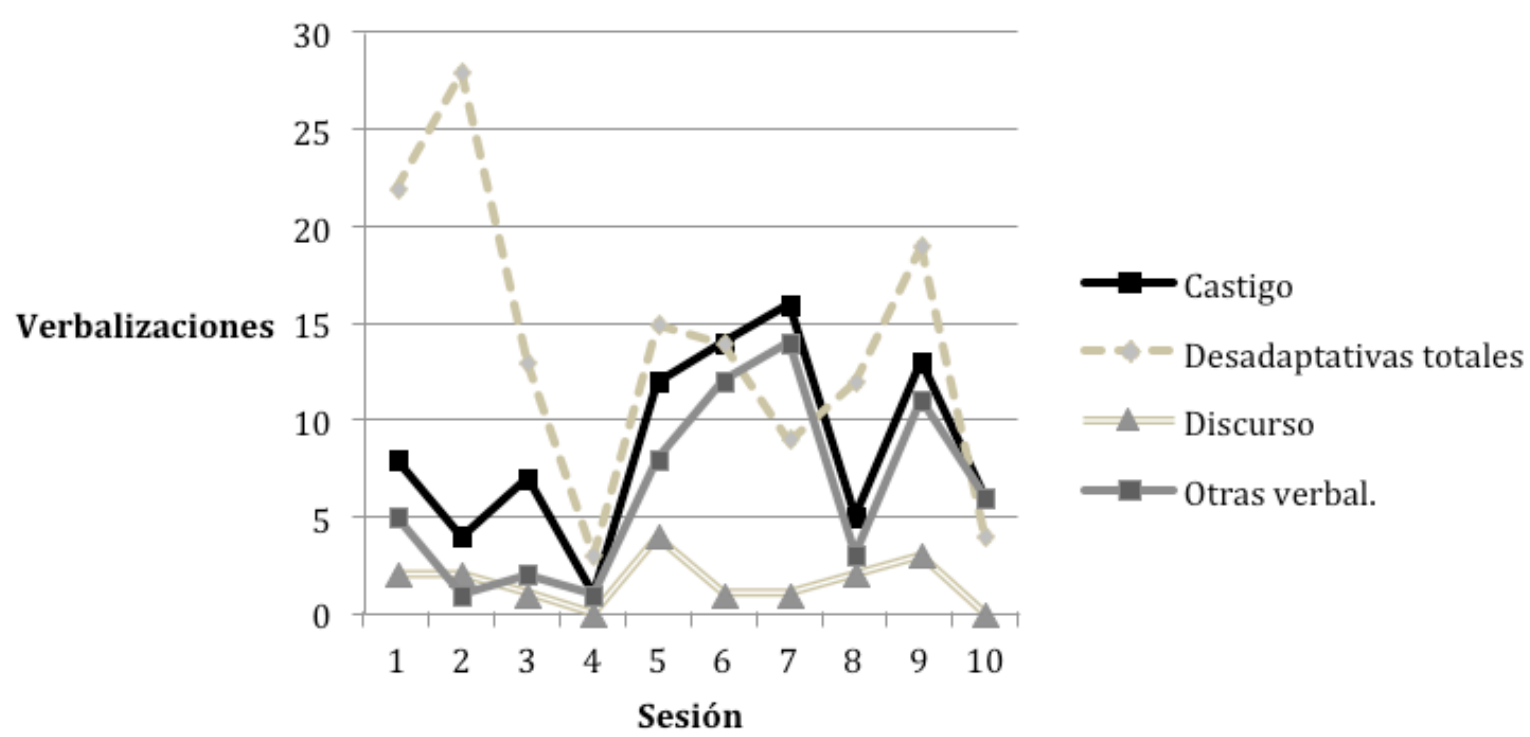

Figura 3. Verbalizaciones de castigo del terapeuta según la definición del SISC-INTER-CVT (Ruiz, 2011) y verbalizaciones del cliente (Frecuencias absolutas). 
SISC-INTER-CVT (Ruiz, 2011)- y compararla con la evolución de las verbalizaciones desadaptativas totales del cliente según el SISC-DESADCVC. Así mismo, se han incluido las categorías del cliente Discurso y Otras Verbalizaciones. Como se puede apreciar, aunque siguen tendencias generales parecidas, no existe una correspondencia total entre las verbalizaciones desadaptativas del cliente y las verbalizaciones de castigo del terapeuta. Por otro lado, como se recoge en la Tabla 2, las verbalizaciones de castigo no se dirigieron siempre a las verbalizaciones con un contenido desadaptativo, tal y como lo hemos definido en este trabajo, sino que se dirigieron sobre todo a otro tipo de contenidos -Otras Verbalizaciones- o a la conducta verbal en sí misma -Discurso-. En la Figura 3 se aprecia claramente la relación entre las verbalizaciones de castigo del terapeuta y la categoría Otras Verbalizaciones. Así pues, ni todas las verbalizaciones desadaptativas del cliente fueron castigadas ni todos los castigos del terapeuta fueron dirigidos a verbalizaciones desadaptativas del cliente, aunque es cierto que puede apreciarse cierta relación entre ellas. Esto tiene sentido por diversas razones que se desarrollan en profundidad en el siguiente apartado. Por el momento, basta con decir que los objetivos del psicólogo cambian a lo largo de la terapia, de tal forma que no siempre busca reducir o eliminar las verbalizaciones desadaptativas del cliente.

\section{Discusión}

Como ya se ha comentado, el trabajo aquí expuesto supone únicamente un primer acercamiento al papel del castigo verbal en el con- texto clínico. Tan sólo se ha llevado a cabo un análisis descriptivo de la evolución de ciertas verbalizaciones del cliente y de su relación con las verbalizaciones punitivas del terapeuta. Sin embargo, son varias las conclusiones que se pueden extraer de los resultados de dicho análisis:

- En primer lugar, los resultados muestran que las verbalizaciones desadaptativas del cliente siguieron una tendencia general descendente a lo largo de la terapia, como era de esperar. Las mayores frecuencias se encontraron en la sesiones de evaluación, para ir reduciéndose hasta prácticamente desaparecer en la sesión de consolidación. Obviamente, no se puede afirmar que esta reducción se deba únicamente a la aplicación del castigo. En la terapia entran en juego muchos otros mecanismos, como la extinción o el reforzamiento diferencial, que también influyen en la reducción de la frecuencia de aparición de verbalizaciones desadaptativas. En este sentido, la disminución o desaparición de las verbalizaciones desadaptativas no responde a la aplicación del castigo por parte del terapeuta, sino a la resolución de aquellos problemas a los que hacen referencia. Una futura línea de investigación puede ir en la dirección de identificar y separar el efecto de cada uno de estos mecanismos en el descenso de las verbalizaciones desadaptativas en terapia.

- Si bien la tendencia general era descendente, un análisis detallado de la evolución de cada una de las categorías contempladas en el SISC-DESAD-CVC, mostró altibajos en la 
frecuencia de algunos tipos de verbalizaciones desadaptativas a lo largo de la terapia. Es decir, si bien se produjo un descenso en la frecuencia de aparición de todas las categorías, este descenso no fue uniforme y sostenido en todas ellas. Así, se encontraron picos y valles en distintos momentos de la terapia para varias de las categorías contempladas en nuestro sistema. Lo que estos altibajos reflejan es el propio trabajo de abordaje clínico de los problemas del cliente. Cada categoría de nuestro sistema hace referencia a un área problema y, en consecuencia, a uno o varios objetivos terapéuticos. Los picos en la frecuencia de aparición de cada categoría reflejan que el cliente y el terapeuta están trabajando sobre esos objetivos. El cliente habla de sus problemas y de las dificultades que tiene a la hora de llevar a cabo las tareas que le plantea el terapeuta para resolverlos. Esto se refleja en un aumento puntual en la frecuencia de aquella categoría del SISCDESAD-CVC que hace referencia al problema que se está trabajando.

- El análisis de la relación entre las verbalizaciones desadaptativas del cliente y las verbalizaciones punitivas del terapeuta mostró que no existe una correspondencia uno-uno entre ambas. Es decir, que ni todas las verbalizaciones desadaptativas del cliente iban seguidas de castigos del terapeuta ni todos los castigos del terapeuta iban precedidos de verbalizaciones desadaptativas del cliente. Veamos más detenidamente lo que implican estos dos hechos. En primer lugar, se pudo observar que el terapeuta no castigaba todas y cada una de las verbalizaciones desadaptativas del cliente.
Es más, un análisis detallado mostró que estas verbalizaciones no sólo no eran castigadas, sino que eran exploradas y reforzadas por el terapeuta en todas las fases de la terapia. Ello se reflejó en que muchas de las verbalizaciones del cliente que se categorizaron como desadaptativas fueron seguidas de verbalizaciones del terapeuta categorizadas como discriminativa o refuerzo según el SISC-INTER-CVT (Ruiz, 2011). Lo que esto podría significar que ese tipo de verbalizaciones no serían anti terapéuticas en el sentido estricto del término, sino justo al contrario, ayudarían al desarrollo de la terapia. El terapeuta buscaría (discriminaría) y reforzaría estas verbalizaciones del cliente porque serían indicadores de aquellos aspectos que son relevantes para trabajar en terapia, permitiéndole planificar adecuadamente las sesiones y hacer hincapié en aquello que es más importante. En este sentido podrían considerarse, incluso, pro terapéuticas. Por otro lado, las observaciones mostraron que el terapeuta castigaba muchas otras cosas a parte de las verbalizaciones que se categorizaron como desadaptativas. Así, se encontró que se castigaban conductas como la de mostrar desacuerdo ante lo que proponía el terapeuta, no contestar a lo que se estaba preguntando, desviarse del tema, interrumpir o expresar interpretaciones inexactas, extremistas o contradictorias sobre el tema de discusión. Retomando lo anterior, todas estas verbalizaciones, sin ser necesariamente desadaptativas, serían anti terapéuticas porque que impedirían el desarrollo óptimo de la intervención. Así pues, el terapeuta se valdría del castigo como recurso para reducirlas o eliminarlas. Estas observa- 
ciones abren un interesante debate sobre las diferencias entre una conducta "desadaptativa" y una conducta "anti terapéutica" y lo que se entiende cuando nos referimos a cada una de ellas. Así mismo, nos muestra un posible camino a seguir en lo que al estudio del castigo verbal en terapia se refiere. $Y$ es que es posible que papel del castigo en clínica no sea el de eliminar las verbalizaciones desadaptativas del cliente, sino el de eliminar aquellas conductas que impiden el adecuado progreso de la terapia en sí misma.

Insistimos en el carácter exploratorio del presente trabajo. Se abre ahora todo un campo de posibilidades de investigación en lo que al proceso de castigo se refiere, particularmente a su aplicación en la práctica clínica. Hace falta seguir investigado cómo y en qué momentos se utiliza el castigo en terapia, así como sus efectos directos e indirectos sobre la conducta del cliente en sesión. Interesa determinar si las verbalizaciones punitivas del terapeuta son realmente castigos en el sentido funcional del término, es decir, si su aplicación a una conducta reduce la probabilidad de que dicha conducta se repita en el futuro. Del mismo modo, sería interesante llegar a manipular el castigo como variable independiente y observar sus efectos en distintas condiciones mediante, por ejemplo, el entrenamiento de terapeutas en distintos estilos de aplicación del castigo en terapia.

\section{Referencias}

Dura, J. R. (1991). Controlling extremely dangerous aggressive outbursts when functional analysis fails. Psychological Reports, 69, 451-459.

Froján, M. X., Calero, A. y Montaño, M. (2011). Study of the socratic method during cognitive restructuring. Clinical Psychology and Psychotherapy, 18, 110-123

Froján, M. X., Montaño, M. y Calero, A. (2006). ¿Por qué la gente cambia en terapia? Un estudio preliminar. Psicothema, 18, 797-803.

Froján, M. X., Montaño, M., Calero, A y Ruiz, E. (2011) Aproximación al estudio funcional de la interacción verbal entre terapeuta y cliente durante el proceso terapéutico. Clínica y Salud, 22, 69-85.

Froján, M. X., Pardo, R., Vargas, I. y Linares, F. (2011) Análisis de las reglas en el contexto clínico. EduPsykhé, 10 (1), 135-154.

Grace, N. C., Kahng, S. W., \& Fisher, W. W. (1994). Balancing social acceptability with treatment effectiveness of an intrusive procedure: A case report. Journal of Applied Behavior Analysis, 27, 171-172.

Hagopian, L. P., Fisher, W. W., Sullivan, M. T., Acquisto, J., \& LeBlanc, L. A. (1998). Effectiveness of functional communication training with and without extinction and punishment. Journal of Applied Behavior Analysis, 31, 211-235.

Iwata, B. A., Pace, G. M., Dorsey, M. F., Zarcone, J. R., Vollmer, T. R., Smith, R. G., et al. (1994). The functions of self-injurious behavior: An experimental-epidemiological analysis. Journal of Applied Behavior Analysis, 27, 215-240.

Johnston, J (1985). Controlling professional behavior: a review of "The effects of punishment on human behavior" by Axelrod and Apsche. The Behavior Analyst, 8 (1), 111-119

Lerman, D. C., \& Iwata, B. A. (1996b). A methodology for distinguishing between extinction and punishment effects associated with response blocking. Journal of Applied Behavior Analysis, 29, 231-233.

Lerman, D. y Vondran, C. (2002). On the status of knowledge for using punishment: im- 
plications for treating behavior disorders. Journal of applied behavior analysis, 35 (4), 431-464

Martin, G., y Pear, J. (1983). Behavior modification: What is it and how to do it. Englewood Cliffs, N.J. Prentice-Hall.

Mazaleski, J. L., Iwata, B. A., Rodgers, T. A., Vollmer, T. R., \& Zarcone, J. R. (1994). Protective equipment as treatment for stereotypic hand mouthing: Sensory extinction or punishment effects? Journal of Applied Behavior Analysis, 27, 345-355.

Ruiz, E. (2011) Aproximación al estudio funcional de la interacción verbal entre terapeuta y cliente durante el proceso terapéutico. Tesis doctoral inédita leída en la Universidad Autónoma de Madrid, Facultad de Psicología, Departamento de Psicología Biológica y de la Salud.

Ruiz, E., Froján, M. X., Calero, A. (2013). Análisis de la conducta verbal del cliente durante el proceso terapéutico. Anales de Psicología.

Vollmer, T. R., \& Iwata, B. A. (1993). Implications of a functional analysis technology for the use of restrictive behavioral interventions. Child and Adolescent Mental Health Care, 3, 95-113.

Wacker, D. P., Steege, M. W., Northup, J., Sasso, G. M., Berg, W., Reimers, T., et al. (1990). A component analysis of functional communication training across three topographies of severe behavior problems. Journal of Applied Behavior Analysis, 23, 417-429. 